\title{
5G-V2X Communications and Networking for Connected and Autonomous Vehicles
}

\author{
Antonella Molinaro ${ }^{1,2}, * \mathbb{D}$, Claudia Campolo ${ }^{1} \mathbb{D}$, Jérôme Härri ${ }^{3}$, Christian Esteve Rothenberg $4{ }^{4}$ \\ and Alexey Vinel ${ }^{5}$ (D) \\ 1 Dipartimento DIIES, Università Mediterranea di Reggio Calabria, Via Graziella, Loc. Feo di Vito, \\ 89122 Reggio Calabria, Italy; claudia.campolo@unirc.it \\ 2 Laboratoire des Signaux et Systémes (L2S), CentraleSupélec-CNRS-Université Paris-Sud, \\ Université Paris-Saclay, 91190 Gif-sur-Yvette, France \\ 3 Department of Communication Systems, EURECOM, 450 Route des Chappes, 06904 Sophia Antipolis, \\ France; Jerome.Haerri@eurecom.fr \\ 4 School of Electrical and Computer Engineering, University of Campinas (UNICAMP), Campinas 13083-872, \\ Brazil; chesteve@dca.fee.unicamp.br \\ 5 School of Information Technology, Halmstad University, 30118 Halmstad, Sweden; alexey.vinel@hh.se \\ * Correspondence: antonella.molinaro@unirc.it
}

Received: 28 June 2020; Accepted: 2 July 2020; Published: 8 July 2020

In the last decade, there has been a surge in interest in connected and automated vehicles (CAVs) and related enabling technologies in the fields of communication, automation, computing, sensing, and positioning. Indeed, CAVs are expected to revolutionize future transportation and quality of life. By leveraging novel efficient air interfaces, a combination of different radio access technologies, and disruptive network softwarization techniques and edge/cloud computing solutions, fifth generation (5G) positions itself to guarantee the ultra-low latency, the ultra-high reliability, and the high-data-rate vehicle-to-everything (V2X) connectivity demanded by CAV applications.

The complexity and uniqueness of the vehicular ecosystem, the variety of involved stakeholders and standards-developing organizations, the evolving nature of envisioned CAV applications, and the numerous activities engaging the research community in 5G-enabled V2X are among the main motivations of this Special Issue aimed at collecting the most recent achievements in this vibrant field.

The papers included in this Special Issue of the Future Internet journal provide a comprehensive overview of the emerging topics in the field of 5G V2X communications and networking. In more detail, they cover aspects ranging from the Third Generation Partnership Project (3GPP) 5G and V2X architectural enhancements to physical layer coding techniques and radio access technologies, while considering issues associated with network orchestration and localization. Some of them also report achievements from recently concluded and ongoing collaborative research projects. Moreover, the collected papers are heterogeneous in nature, which is one of the main strengths of the Special Issue: they range from technically sound theoretical analysis and timely architectural design contributions to algorithmic design and practical contributions.

In particular, a total of six excellent original research papers, with authors from both industry and academia, have been accepted that address many of the aforementioned challenges, following a rigorous review process involving expert reviewers in the respective areas.

The first paper [1] dissects the main challenges associated with cross-border and multi-operator V2X deployment focusing on three representative Cooperative, Connected, and Automated Mobility (CCAM) services: Tele-Operated Driving, High-Definition map generation and distribution for autonomous vehicles, and Anticipated Cooperative Collision Avoidance. Enhancements of roaming schemes, inter-edge-cloud coordination mechanisms, network slice selection, and Quality of Service 
(QoS) prediction are presented as solutions conceived within 5GCroCo, which is a Horizon 2020 EU project in which $5 \mathrm{G}$ technologies will be trialed for CCAM in cross-border scenarios.

The achievements of a further Horizon 2020 project, 5G Communication Automotive Research and innovation (5GCAR), are reported in the second paper [2]. The authors present the enhancements-e.g., in terms of network orchestration and management, edge computing, and multi-connectivity cooperation-conceived by 5GCAR for the 5G system level architecture proposed by the 3GPP to support V2X use cases.

By focusing on the 3GPP architecture for V2X communications, the third paper [3] focuses on the networking aspects and describes the potential for leveraging Information-Centric Networking, and Named Data Networking (NDN) in particular, for data exchange in V2X contexts. The goal of the paper is to analyze the specific design aspects and enhancements in the 3GPP V2X architecture to enable NDN-based data exchange and relevant features (e.g., in-network caching and the aggregation of named requests) and to assess the achieved improvements over traditional host-centric IP networking.

Departing from the architectural design of V2X communications-related aspects, the fourth paper [4] provides an overview of the status quo and of the ongoing evolution of the two most prominent radio access technologies for V2X, and vehicle-to-vehicle communications in particular, i.e., IEEE 802.11p and Cellular-V2X. A comprehensive evaluation study is conducted through large-scale simulations to shed light on the advantages and drawbacks of the two technologies and to pinpoint the main conditions and parameters that affect their performance.

The fifth paper [5] targets the improvements of the reliability of vehicle-to-roadside connectivity when the User Datagram Protocol (UDP) is used to satisfy real-time requirements, by introducing redundancy into the transmitted data. To this aim, the authors propose an inter-packet error control coding scheme to improve the recovery of data with a focus on convolutional coding techniques. Simulations for the memoryless binary erasure channel, channels with memory, and IEEE 802.11p radio are provided.

Moving on to more practical implementations, the authors of the sixth paper [6] investigate the usage of Long Range Wide Area Network (LoRaWAN) technology to geolocalise vehicles indoors and counteract the well-known limitations of the Global Navigation Satellite System (GNSS). In particular, the proposal achieves the localisation of a vehicle within a 20-30 m range with a low deployment cost, by leveraging the estimation of the behaviour of a LoRaWAN channel established between simple client devices and LoRaWAN gateways, and using trilateration.

Funding: EURECOM acknowledges the support of its industrial members, namely, BMW Group, IABG, Monaco Telecom, Orange, SAP and Symantec.

Acknowledgments: It has been a great pleasure to put together this Special Issue and gather interesting research papers, which provide and assess novel ideas and solutions in the field of 5G V2X communications and networking. We are extremely grateful to Dino Giuli, the Editor-in-Chief of the Future Internet journal, for the opportunity to organize this Special Issue and to all the managing Editors supporting us. We thank all the authors for submitting their papers to this Special Issue and all the volunteer reviewers for their hard work in evaluating these submissions within the tight deadlines and for their valuable remarks that have contributed to the quality of the published articles.

Conflicts of Interest: The authors declare no conflict of interest.

\section{References}

1. Kousaridas, A.; Schimpe, A.; Euler, S.; Vilajosana, X.; Fallgren, M.; Landi, G.; Moscatelli, F.; Barmpounakis, S.; Vázquez-Gallego, F.; Sedar, R.; et al. 5G Cross-Border Operation for Connected and Automated Mobility: Challenges and Solutions. Future Internet 2019, 12, 5. [CrossRef]

2. Condoluci, M.; Gallo, L.; Mussot, L.; Kousaridas, A.; Spapis, P.; Mahlouji, M.; Mahmoodi, T. 5G V2X System-Level Architecture of 5GCAR Project. Future Internet 2019, 11, 217. [CrossRef]

3. Amadeo, M.; Campolo, C.; Molinaro, A.; Härri, J.; Rothenberg, C.E.; Vinel, A. Enhancing the 3GPP V2X Architecture with Information-Centric Networking. Future Internet 2019, 11, 199. [CrossRef] 
4. Bazzi, A.; Cecchini, G.; Menarini, M.; Masini, B.M.; Zanella, A. Survey and Perspectives of Vehicular Wi-Fi versus Sidelink Cellular-V2X in the 5G Era. Future Internet 2019, 11, 122. [CrossRef]

5. Bocharova, I.; Kudryashov, B.; Lyamin, N.; Frick, E.; Rabi, M.; Vinel, A. Low Delay Inter-Packet Coding in Vehicular Networks. Future Internet 2019, 11, 212. [CrossRef]

6. Manzoni, P.; Calafate, C.; Cano, J.C.; Hernandez-Orallo, E. Indoor Vehicles Geolocalization Using LoRaWAN. Future Internet 2019, 11, 124. [CrossRef]

(C) 2020 by the authors. Licensee MDPI, Basel, Switzerland. This article is an open access article distributed under the terms and conditions of the Creative Commons Attribution (CC BY) license (http://creativecommons.org/licenses/by/4.0/). 